\title{
Reactive Arthritis The Reiter-Fiessinger-Leroy Syndrome
}

\author{
DOINA CARINA VOINESCU1, ADRIAN BEZNEA ${ }^{1 *}$, CIPRIAN DINU ${ }^{1}$, CAMELIA ANA GRIGORE ${ }^{1}$, \\ SILVIA FOTEA ${ }^{*}$, CRISTINA PREDA ${ }^{2 *}$, MIHAELA-ELENA ZAMFIRESCU ${ }^{3}$, DANIELA LAURA BURUIANA ${ }^{1}$, \\ AUREL NECHITA ${ }^{1}$ \\ ${ }^{1}$ Dunarea de Jos University of Medicine and Pharmacy, 47 Domneasca Str., 800008, Galaţi, Romania \\ 2"Grigore T. Popa" University of Medicine and Pharmacy, 16 Universitatii Str., 700115, Iasi, Romania \\ 3"Ovidius" University of Medicine and Pharmacy, Faculty of Medicine, 1 Universitatii Alley, 900470, Constanta, Romania
}

The role of creating the expression of reactive arthritis rests with AHO in 1973, which defines reactive arthritis as an acute, unsupportive, sterile inflammatory arthropathy, arising from infections located at a distance in people with a certain genetic predisposition; the disease is systemic and, despite its name, is not limited to the joints. Reactive arthritis (RA), included in Reiter syndrome (with the classic triad of urethritis, conjunctivitis and arthritis), is a non-suppurative inflammatory joint pathology, with a minimal autoimmune component, which it is installed following intestinal or urogenital infections, mainly in people with genetic predisposition. Reactive arthritis is a form of arthritis that affects the joints, urethra, eyes and membranes and sometimes the skin and mucous membranes. This condition is also called Reiter's syndrome, although this name only indicates a subtype. Reactive arthritis is a seronegative spondyloarthropathy, which brings together a group of disorders that cause inflammation in the body, especially in the spine - such as ankylosing spondylitis, and in the legs. The study covers a number of 20 patients (11 men, 9 women) admitted to the Rheumatology Service of the Clinical Recovery Hospital, between 2010 and 2011, aged over 20, who were diagnosed with reactive arthritis. The existence of a structural similarity between the etiological agent and the HLA-B27 molecule determines either the recognition of the disease-causing germ as a self, so that no immune response will be initiated against it and the antigen will be able to trigger the disease, or the recognition of the germ as nonself, with the triggering of an immune response aimed at both itself and against the HLA-B27 molecule carrying cells of similar structure. Reactive arthritis (RA), included in the Reiter syndrome (with the classic triad of urethritis, conjunctivitis and arthritis), is a non-suppurative inflammatory joint pathology, with a minimal autoimmune component, which is installed as a result of intestinal or urogenital infections, mainly in people with genetic predisposition.

Keywords: reactive arthritis, infections, Reiter's syndrome, joint pathology, seronegative spondyloarthropathy, thyroiditis

Reactive arthritis refers to acute non-purulent arthritis, complications of an infection with any localization in the body; in the last years the term has been used especially for spondyloarthropathies that occur as a result of enteral or urogenital infections and found predominantly in individuals with HLA B27 histocompatibility antigen. The constellation of clinical manifestations often known as Reiter syndrome can also be included. Other forms of reactive arthritis are not associated with HLAB27 and represent a different spectrum of clinical data such as acute joint rheumatism.

Reactive arthritis is defined as the arthritis that appears 1-4 weeks after an enteral or urogenital infection, especially in people with HLA B27. It is characterized by two particular aspects: it is different from infectious arthritis because in the case of reactive arthritis the culture of viable bacteria in the joint has not been obtained and there is reactive arthritis that cannot be classified as seronegative spondyloarthropathy because it has another entry and is not associated with HLA B27, arthritis from acute joint rheumatism (the arthritis reactive to streptococcal infection). Currently, reactive arthritis is considered only arthritis that can be classified as seronegative spondyloarthropathy; it is part of the group of spondylarthritis, those rheumatic diseases characterized by the absence of the rheumatoid factor; an inflammatory peripheral arthropathy affecting the sacroiliac joint and the possibility of extra-joint clinical manifestations; the association of this disease with genetic factors, especially related to the presence of AgHLA-B27.

The condition has been widely discussed in terms of definition, etiopathogenesis and clinical appearance. The major clinical element "reactive and aseptic arthritis" must be understood on the basis of three fundamental ideas for its clear definition: the appearance after a variable interval (5-12 days) from the infection; the negativity of the cultures in the articular fluid (the presence of bacterial antigens in the joints has recently been demonstrated); the absence of the immediate response to antibiotic therapy.

\footnotetext{
*email: adrianbeznea@yahoo.com; silvia.fotea@ugal; cpredal@yahoo.com
} 
Epidemiology often affects the young adult and equally affects both genders. The annual incidence is of 30 40/100,000 inhabitants. Genetic association, family incidence, HLA-B27. It is frequently associated with various infections.

The pathogenesis of reactive arthritis (RA) is complex, involving a number of genetic, immune, environmental factors that mingle and generate articular and extra-articular inflammatory phenomena.

The etiologic agent is possibly involved at both levels, joint and entrance gate, as well as indirectly, by studying the specific humoral immune response. Despite the fact that the articular fluid is sterile in reactive arthritis, the harvesting being negative, a persistence of the bacterial components at the articular level, or at the entrance gate level has been suspected from the beginning.

The CLI monoclonal antibodies of synovial biopsy and cells of the articular fluid study revealed the major component of the outer membrane of Chlamydia Trachomatis and the lipopolysaccharides of Chlamydia and Yersinia Enlerocolitica. Analogously Salmonella Ag was detected by rabbit Ab, produced against the bacteria, and by monoclonal Ab specific for lipopolysaccharide.

The characteristic of the humoral response to arthritis-causing microorganisms is the increased level of Ab and their prolonged persistence at this level, which could mean continuous antigenic stimulation. The antibodies present are frequently of the IgM type, which can also be explained by the fact that they have a short life and disappear when arthritis develops, especially when the latency interval is greater than one week. The entry gate (digestive, genital, and respiratory) of the infectious agent may represent the initiation of the pathogenic process of reactive arthritis.

The identification of bacterial species capable of clinically inducing and the discovery that up to three quarters of patients possess HLA B27 antigen led to the formulation of the concept of RA as a clinical syndrome that appeared in a host organism with genetic susceptibility and initiated by a specific etiological agent.

Of the three entry gates, a special importance has been given to the intestine due to the numerous germs that act at this level, as well as due to its presumedly pathogenic role in other spondylarthritis as well. All etiologic agents with digestive entry gate (Enterobac-teriacee-Yersinia, salmonella, Shigella, Campylobacter jejuni), viruses, protozoa (Giardia, Entamoeba) have a local enteroinvasive action mechanism, with the penetration and multiplication in the intestinal mucosa, where they determine destructive lesions of variable intensity. This mechanism is the only one that allows and explains the subsequent diffusion of endotoxins through the lymphatic and blood pathways throughout the body, the endotoxin that results from the interaction of the local defence agent (specific and non-specific). This explains the humoral response in IgA of certain reactive arthritis. Increased IgA corresponds to a specific antibacterial response and is a prognostic element for numerous reactive arthritis: a persistent anti-Yersine IgA response is associated with severe and chronic post-Yersinian reactive arthritis. These IgA participate in the formation of immune complexes responsible for numerous articular or systemic manifestations.

The two subclasses of $\operatorname{IgA}$ (A-1 and A-2) are produced in variable proportions in patients with inflammatory bowel disease. In inflammatory lesions, an increase of IgA-1 synthesis, a reduction of IgA-2 and a decreased expression of chain I can be observed. The protective action of $\operatorname{IgA}$ at the mucosal level is dependent on the presence of the secretory component, which gives the dimer molecular stability and resistance to endoluminal proteolytic digestion.

The reduction of IgA-2 synthesis in inflammatory bowel disease as well as decreased expression of the $\mathrm{J}$ chain are responsible for the poor protection of the intestinal mucosa by secretory IgA, which favours inflammatory lesions.

The pathogenic role of genetic factors is major in reactive arthritis and is demonstrated by the increased frequency of Ags of the major histocompatibility complex. For specific, typical reactive arthritis, AgMHC of class I-HLA B27 is involved and for atypical ones (RAA, Lyme disease), AgMHC of class II-HLA DR2, DR3, DR4.

An important pathogenic role would be held by position 45 of the $\alpha 1$ chain of antigen B27, a position designated as "exogenous antigen binding site". It has a unique structure in the HLA-B27 molecule, common to all subtypes, and it would be normal for the antigenic peptide chain that is attached here to be unique as well.

Two factors are involved in the occurrence of reactive arthritis: genetical and infectious.

The genetic factor is HLA B27. Another gene that is involved in the occurrence of reactive arthritis is TAP (transporter associated with antigen processing). The infectious factor consists of germs that share the same characteristics: they have the ability to synthesize the lipopolysaccharide, adhere easily to the cell membrane, and invade the cells of the intestinal or urogenital mucosa, persisting intracellularly.

Among the enteric bacteria we mention: Shigella flexuri, Salmonella, Campylobacter, Yersinia. The urogenital bacteria involved in the occurrence of reactive arthritis are: Chlamydia Trachomatis, Ureaplasma urealyticum.

The manifestations of reactive arthritis include: inflammatory arthritis of the large joints (often affecting the spine); ocular inflammation (conjunctivitis or uveitis); urethritis in men or cervicitis in women; another common manifestation in this disease is the skin lesions that resemble psoriasis. This includes balanitis circinata and keratoderma blennorrhagicum. 
The symptoms of reactive arthritis may begin 1-3 weeks after infection and may last 3 to 12 months, varying in severity. The first symptoms that appear are the urinary ones, followed by fever and symptoms of the eyes; however, this order will not be followed in all cases and not all types of symptoms may occur:

Urethritis, inflammation of the tube that connects the bladder and the outside of the body (urethra); urethritis leads to leaks that can be seen on the head of the penis or in the vaginal area; it also causes pain or burns when urinating. Men may have inflammation of the prostate (prostatitis), and women may have inflammation of the cervix (cervicitis).

Conjunctivitis or uveitis, inflammation of the thin membrane covering the eyeball and eyelid lines (conjunctiva), or of the inner eye (uveitis); conjunctivitis causes redness and itching; uveitis is much more severe and causes pain, blurring of vision and sensitivity to light, as well as redness of the eyes.

Arthritis, pain, redness and swelling that usually affect the knees, ankles and legs; pain often occurs in the heel; it can cause swelling of the fingers and toes, often associated with lower back and buttocks pain; it can cause spondylitis (inflammation of the joints between the vertebrae of the spine).

Reactive arthritis may also be associated with skin symptoms, including oral ulcers, rashes or swelling on the soles or palms, and painless penis lesions. The disorder is most commonly seen in men under the age of 40, although women may also have reactive arthritis. Most people with reactive arthritis have a gene that makes them susceptible, called HLA-B27.

After about 1-4 weeks from onset, systemic, articular and extra-articular manifestations may occur. Systemic manifestations consist of altered general condition, fatigue, weight loss, anorexia, fever up to 39 degrees.

The articular manifestations are: peripheral arthritis, as this can be asymmetrical mono-articular or oligoarticular and affects the large joints of the lower limbs (knee, ankle); it is acute, migratory being accompanied by signs of inflammation. Peripheral arthritis can also be chronic, but much less frequent and affects the small or upper joints or those at the upper limbs level; axial arthritis: sacroiliitis (expressed by pain in the buttocks) accompanied by spondylitis (back inflammation); enthesitis-thalalgia (Achilles tendonitis) chest pain (inflammation of the insertion of intercostal muscles on the ribs), pain in the iliac crest; dactylitis: swelling of a finger (synovitis, periostitis), muscular hypertrophy in the quadriceps and rapidly occurring in knee arthritis.

Extra-articular manifestations may be cutaneous-mucosa (keratoderma blenorrhagicum - manifested by clear vesicles on erythematous background with localization in the palms and plants). Another manifestation is circinate balanitis (superficial ulceration, painless irregular border with localization on the glans or the balanopreputial canal). Painful ulceration may also occur in the digestive tract (oral cavity).

Sterile urethritis may be present in the reactive arthritis, through an immune mechanism. In arthritis determined by Yersinia, nodes erythema may occur.

Other extra-articular changes are those of the nail (colour changes, hyperkeratosis, onycholysis similar to those of psoriasis), ocular (conjunctivitis and anterior uveitis). Cardiac manifestations occur in less than 10\% being the consequence of inflammation of the root of the aorta causing atrioventricular conduction disorders.

Renal manifestations such as mesangial glomerulonephritis with Ig A deposits, amyloidosis, can also be encountered.

The neurological manifestations associated with reactive arthritis may be peripheral neuropathy, transverse myelitis, encephalitis.

The positive diagnosis is based on clinical examination, history and laboratory tests, the presence of inflammatory syndrome and positive serology.

The general clinical picture of RA is polymorphic and varies depending on the class of arthritis, the route of transmission (digestive, urogenital, respiratory) and shows certain variations depending on the particular clinical forms of RA, as well as on the etiological causative agent.

As a clinical element of great importance for diagnosis, the onset of these arthritis should be specified, namely 1-4 weeks after a digestive or urogenital infection; it may be closer to the infectious episode (3-6 days), when arthritis is acute, with major inflammatory phenomena and accompanied by fever.

The hematological examination shows anemia and leucocytosis, and the acute inflammation tests are positive, VSH, fibrinogen, C-reactive protein-Xi antitrypsin, complement, supporting the possibility of inflammatory rheumatism.

The exploration of the dysimmune syndrome with the determination, in particular, of immunoglobulins, of circulating immune complexes and cryoglobulins converges to the idea of arthritis reactivity, with hyperimmunoglobulinemia being frequent, especially $\mathrm{A}$ and $\mathrm{G}$ and, more rarely, hyper- $\operatorname{IgM}$, a recent infection control.

The complementary serological examinations, meant to establish a differential diagnosis, are the Latex and WaalerRose reactions, frequently negative (in some viral arthritis these can be positive); uric acid, anti-streptolysin titre 0 , liver samples, determination of HBs antigen.

Paraclinical examinations that acquire some specificity for RA (correlated with the patient's infectious history) conduct to the idea of isolating the infectious agents at the entrance gate and are represented by pharyngeal and nasal exudates, sputum examination, stool culture, urocultures, hemocultures and urethra secretion test. 
Determination of HLA-B27 antigen, found in $60-85 \%$ of patients, is of limited use in the diagnosis of RA, since some of the patients (15-40\%) do not have this antigen, and of the subjects who possess HLA-B27 antigen (in the general population), only a small part can have RA. However, the presence of HLA-B27 antigen increases the probability of RA diagnosis. The positivity of this antigen, on the other hand, has a prognostic value, expressing the tendency of chronicization, the high incidence of ocular manifestations and the risk of evolution towards an ankylopoietic spondylitis.

The examination of synovial fluid that highlights a non-specific inflammatory aspect is useful in the differential diagnosis with septic arthritis and microcrystalline arthropathy.

\section{Reactive arthritis - Reiter syndrome interrelation}

Reiter-Fiessinger-Leroy syndrome is characterized by conjunctivitis, urethritis, arthritis, which often follows dysentery, and may have various clinical pictures.

It is called reactive arthritis and is known as Reiter's syndrome, because it is considered to be an inflammatory disorder following a bacterial infection in the intestinal or urogenital level. Often, people who experience pain in the joints of the body and with inflammatory processes do not suspect even for a moment that the condition they suffer from is a direct consequence of a sexually transmitted disease.

The bacterial structures of Sallmonela, Yersinia, Chlamydia have been successfully identified at articular level, but the identification of this bacteria through harvesting from the joint liquid has not been accomplished. The clinical manifestations, relatively similar to those of positive HLA-B27 reactive arthritis, and its similar frequency in their pathogenesis, prompted many authors to consider Reiter Syndrome a RA with a generally noisier clinical picture. Two forms of Reiter syndrome are described based on the conditions of occurrence: endemic, often following urethritis; epidemic following dysentery. The general symptoms commonly encountered in patients with Reiter syndrome are: fever, asthenia, tiredness, loss of appetite, weight loss and disorder of other systems and organs, resulting in complications, sometimes severe.

Reactive arthritis associated with HLA-B27 - These are acute, non-suppurative and sterile inflammatory arthropathies that occur at a distance in terms of time and place from a primary infectious outbreak located in the digestive or urogenital tract. The microorganisms recognized in the etiology of this arthritis are Yersiria, Shigella, Salmonella, Campylobacter and Clamydia but numerous other enteric or sexually transmitted microorganisms are likely to be involved in the etiology of this arthritis.

Arthritis from HIV infection - HIV-positive patients often have arthritis with an evolution into two forms: a severe cumulative form, the intensity of which increases in a few weeks, or months; a form with relapses and intermittent remissions.

The first form is characterized by widespread but asymmetric polyarticular involvement, joint swelling, erosions and juxta-articular osteoporosis. The intermittent form is characterized by oligoarthritis (knees and/or ankles) with an evolution usually similar to Reiter syndrome from HIV-negative persons. These pathogenic hypotheses have important therapeutic consequences (grouped in three directions): anti-inflammatory therapy; anti-infectious therapy; immunomodulatory therapy.

Reactive arthritis from acute articular rheumatism (AAR)

AAR, this inflammatory, autoimmune disease attributed to antigens common to streptococcus, heart tissue and joint capsule usually occurs 1-5 weeks after streptococcal pharyngitis. The onset of AAR can be brutal if the first manifestation is acute arthritis or insidious, if the initial manifestation is cardiac. Carditis reflects inflammatory lesions in the endocardium, myocardium and pericardium. Carditis, polyarthritis, marginalized erythema, subcutaneous nodules are major manifestations of the disease.

\section{Arthritis from Lyme disease}

Lyme disease caused by Borrelia burgdorferi infection through the bite of infected Ixodes ticks, has a polymorphic clinical picture, consisting of skin, neurological, cardiac and joint changes, developed in three stages.

The treatment of $R A$ is complex, prophylactic and curative, aiming to prevent the occurrence of the disease or its relapses, to shorten the duration of the evolutionary outbreaks and to prevent the chronicization and the evolution towards ankylosing spondylitis.

The curative treatment consists of the administration of anti-inflammatory non-steroidal drugs (NSAIDs), giving priority to indomethacin ( $75-150 \mathrm{mg} /$ day) and phenylbutazone (200-400 mg/day), but also to the specific COX2 inhibitors of the coxib type (rofecoxib, celecoxib).

The underlying treatment is for forms of RA that do not respond or are insufficiently responsive to anti-inflammatory treatment and which tend to become chronic. 
Sulfasalazine (SSZ), a synthetic chemotherapeutic, has a real benefit in RA, as demonstrated by its efficacy in other spondylarthritis (ankylosing spondylitis, psoriatic arthropathy), as well as by several direct studies on RA and Reiter syndrome; early administration seems to be a good choice for AR presumed to have enteric origin. Also, sulfasalazine has a remarkable benefit in RA from HIV infection.

The main mechanisms of action of SSZ are: the real anti-inflammatory effect, resulting from inhibition of lipoxygenase and thromboxane synthetase (Stenson, 1983, 1992); reduction of leukocyte mobility (Rhodes, 1981) and of the activity of proteolytic enzymes (Campbell, 1971); inhibition of IgE-mediated mast cell degranulation (Barrett, 1985);

Methotrexate (MTX) is administered in severe forms of Reiter syndrome and reactive arthritis that have been chronic or have a tendency to develop towards ankylopoietic spondylitis.

Known as antifolic cytostatic, MTX has its own anti-inflammatory (reduces cell infiltration) and immunomodulatory role (still controversial by some researchers).

Anti-infectious therapy is currently justified after demonstrating the persistence of bacterial antigens in inflammatory synovial tissue.

Balneophysiotherapy and kinetotherapy are applied outside the activation spurts, with an anti-allergic, antiinflammatory purpose, for regaining joint mobility and muscle strength.

\section{Experimental part}

\section{Material and method}

The study includes a number of 20 patients (11 men, 9 women) hospitalized in the Rheumatology Service of the Galati Recovery Hospital, between 2010 and 2011, over 20 years old, who were diagnosed with reactive arthritis; the treatment for reactive arthritis in all 20 cases consisted in symptom relief by administration of a medication that includes the administration of corticosteroids, antibiotics, nonsteroidal inflammatory drugs or immunosuppressive drugs.

\section{Results and discussions}

The factors involved in the determinism of reactive arthritis are rheumatic diseases associated with the infectious states. The relationship between infection and arthritis is indirect and is characterized by: the onset of arthritis is 3-4 weeks away from the infectious episode; the articular fluid is aseptic; there is a particular genetic field.

The clinical picture is represented by simple inflammatory arthralgia and/or true arthritis; but the most common is the subacute onset with moderate inflammatory changes.

Reactive arthritis is generally self-limiting, but there is also the possibility of recurrences and forms evolving on a particular genetic field that may evolve to Reiter syndrome and ankylopoietic spondylitis.

The pathogenic hypotheses generate important therapeutic lines, grouped in two attitudes, anti-infectious therapy and immunomodulatory therapy.

Regarding the pathogenic mechanism of reactive arthritis, bacteria are thought to survive in the digestive mucosa and lymph nodes and are subsequently transported via the mononuclear cells to the synovial. At this level, molecular biology techniques have highlighted peptide antigens and nucleic acids from the incriminated bacteria, which together with the immunogenetic properties of the host determine the occurrence of reactive arthritis triggered by the infection.

Antimicrobial treatment, given early and prolonged, specific to the infectious agent, associated with the treatment with non-steroidal anti-inflammatory drugs (NSAIDs) can induce long remissions and even cure the disease. There are certain genetic markers, which are more common in patients with reactive arthritis than in the normal population. For example, the HLA-B27 gene is commonly found in patients with reactive arthritis. Even in patients who have the genetic background that makes them prone to developing reactive arthritis, however, exposure to certain infections appears to be necessary to trigger the onset of the disease.

\section{Conclusions}

In most cases, infections begin in the urinary or genital tract, and the onset of symptoms is caused by the bacterium Chlamydia trachomatis, an infection transmitted through sexual contact. The initial infection can also occur in the digestive system. In these cases, the infection occurs after the consumption of food contaminated with the Salmonella, Yersinia or Campylobacter bacteria.

\section{References}

1.ANANDARAJAH, A., RITCHLIN, C. T., Treatment update on spondyloarthropathy. Curr. Opin. Rheumatol. ,May 2005; 17(3): 247-56.2.

2.CARTER, J. D., VALERIANO, J., VASEY, F. B., Doxycycline versus doxycycline andrifampin in undifferentiated spondyloarthropathy, with special reference to chlamydia-induced arthritis. A prospective, randomized 9-month comparison. J. Rheumatol., Oct 2004; 31 (10): 1973-80.3.

3.CRISTESCU, V., ROMILA, A., MACOVEI, L.A. Polymyalgia Rheumatica - a Disease of the Elderly. 2018 Rev.Chim., 69, no.1, 2018, p. 152 
4.EBERL, G., STUDNICKA-BENKE, A., et al. Development of a disease activity index for theassessment of reactive arthritis (DAREA). Rheumatology. 2000; 39: 148-155.5.

5.FAN, P.T., YU, D. T., Reiter's syndrome. In:Kelley's Textbook of Rheumatology. 2001:1039-67.6.

6.GOTTENBERG, J.E., MERLE-VINCENT, F., BENTABERRY, F., et al. Anti-tumor necrosis factor alpha therapy in fifteen patients with AA amyloidosis secondary to inflammatory arthritides: a followup report of tolerability and efficacy. Arthritis Rheum 2003; 48:2019.

7.HAZENBERG, B.P., VAN RIJSWIJK, M.H., Where has secondary amyloid gone? Ann Rheum Dis 59:577., 2000.

8.IONESCU, R., Spondilita anchilozantă în Esenţialul în Reumatologie sub redactia R. Ionescu, Ed a II-a, Bucuresti 2007.

9.KUNUNEN, M., KOVERO, O., WENNEBERG, B., KONTTINEN, Y. T., Radiographicsigns in the temporo-mandibular joint in Reiter's disease. J. Orofac. Pain., 2002; 16(2):143-7.7.

10.KVIEN, T. K., GASTON, J. S., BARDIN, T., et al. Three month treatment of reactivearthritis with azithromycin: a EULAR double blind, placebo controlled study. Ann. Rheum. Dis., Sep 2004; 63(9): 1113-9.8.

11.LANJEWA, R.D.N., BHOSALE, A., IYER, A., Spectrum of dermatopathologic lesionsassociated with HIV/AIDS in India. Indian J. Pathol. Microbiol., Jul 2002; 45(3): 293-8.9.

12.LEE, A.T., HALL, R.G., PILE, K.D., Reactive joint symptoms following an outbreak of Salmonella typhimurium phage type 135a. J. Rheumatol., Mar 2005; 32(3): 524-7.10.

13.MANDACHE, E., GHERGHICEANU, M., Histopatologia si diagnosticul amiloi-dozei Ed Universitara "Carol Davila" Bucuresti 2006.

14.MACOVEI, L.A., DEBITA, M., ILIE, M., MOISEI, M., CHISCOP, I., CARDONEANU, A., ARBUNE, M, REZUS, E. The role of antioxidative enzyme mechanisms and of oxygen free radicals in reumatoid inflammatory processes. Rev.Chim., 66, no.10, 2015, p. 1645

15.MACOVEI, L.A., MATEI, M.N., NICHITA, A., REZUS, E., LEATA, R., CHISCOP, I., ILIE, M., ARBUNE, M., REZUS, E. Peroxide radicals implications in the inflammatory rheumatic disease. Rev.Chim., 66, no.9, 2015, p.1516

16.MACOVEI, L.A., BURLUI, A., REZUS, E. Biochemical Markers of Bone Turnover in Rheumatoid Arthritis Patients Treated with Glucocorticoids. Rev.Chim., 70, no.2, 2019, p. 623

17.MACOVEI, L.A., CARDONEANU, A., BURLUI A., REZUS, E. Etiopathogenic mechanisms of tobacco constituents in rheumatoid arthritis. 2019 Romanian Journal of Rheumatology 28 (1), pp. 5-9.

18.MACOVEI, L.A., REZUS, E. Cervical spine lesions in rheumatoid arthritis patients. 2016 Revista medico-chirurgicală a Societăţii de Medici şi Naturalisti din Iasi 120 (1), pp. 70-76. PMID: 27125075

19.PETERSEL, D.L., SIGAL, L.H., Reactive arthritis. Infect. Dis. Clin. North Am., Dec 2005; 19(4): 863-83.15.

20.RESNIK, D., Reiter's syndrome. In: Diagnosis of Bone and Joint Disorders. Vol 2.2002:1110.16.

21.REVEILLE, J.D., ARNETT, F.C., Spondyloarthritis: update on pathogenesis andmanagement. Am. J. Med., Jun 2005; $118(6)$ : 592-603.17.

22.ROHEKAR, S., TSUI, F.W., TSUI, H.W., XI,N., RIARH, R., BILOTTA, R., Et al. Symptomatic acute reactive arthritis after an outbreak of salmonella. J. Rheumatol., Aug 2008; 35(8): 1599-602.18.

23.TOWNES, J.M., DEODHAR, A.A., LAINE, E.S., SMITH, K., KRUG, H.E., BARKHUIZEN, A., et al., Reactive arthritis following cultureconfirmed infections withbacterial enteric pathogens in Minnesota and Oregon: a population-based study. Ann. Rheum. Dis., Feb 13 2008.19.

24. Van der HEIJDE, D., SCHIFF, M. H., SIEPER, J., et al., Adalimumab effectiveness for the treatment of ankylosing spondylitis is maintained for up to 2 years: lon- term results from the ATLAS trial, Ann Rheum. Dis 2009.

$\overline{\text { Manuscript received: 07.02.2020 }}$ 